\title{
INVESTIGATION OF A NEW HOVERING AUTONOMOUS UNDERWATER VEHICLE FOR UNDERWATER MISSIONS
}

Submitted: 15th July 2021; accepted: 6th September 2021

\section{Faryar Shamshiri Amirkolai, Reza Hasanzadeh Ghasemi}

DOI: 10.14313/JAMRIS/2-2021/8

\begin{abstract}
It is impossible to implement very tasks by diver, because of complexity of underwater environment and high pressure in the deep sea. These tasks can just be done by a vehicle that includes all special requirements such as: high maneuverability, precise controllability, and especially hovering capability. Underwater robots are integral parts of the industry and marine science. The application of the underwater vehicles has increased with the development of the activities in deep sea. This paper presents a special Hovering type Autonomous Underwater Vehicle (HAUV) for underwater missions. To provide the most suitable and efficient formation of vehicle thrusters for reduction of complexity of control strategies and control of the most degrees of freedom, in this paper, a new thrusters' configuration is investigated, in terms of number of the thrusters, position and the thrust direction of the thrusters. The state feedback controller is designed according to the linear dynamic model and then applied to the non-linear model to validate the controller performance. Designed controller consists of three controllers: horizontal plane controller, vertical plane controller and surge controller. The last controller is developed to control the forward speed. For examination of the system behavior in presence of environmental disturbance and hydrodynamic coefficients uncertainty, the robustness of controller is also investigated.
\end{abstract}

Keywords: Hovering type Autonomous Underwater Vehicle (HAUV); Inspection; State Feedback Controller

\section{Introduction}

Underwater inspection and examination is an essential task for maintenance and diagnosis of damage of underwater structures such as: ship, submarine and subsea pipelines.

The remote inspection of ship hulls and marine structures has become a major concern to operators of vessels and ports, because of the vulnerability of such sites to relatively small mines or other devices placed to destroy or disrupt naval and commercial activities [1]. This has become extremely critical with the threat that ships entering ports and harbors for commerce may serve as carriers of explosives, deadly chemicals and other hazardous materials, with mass destruction in highly populated cities, national landmarks, and other drastic damages at the nation scale as potentially target activities [2].
One approach for underwater inspection is to use of a number of divers. This approach is time-consuming, dangerous and non-efficient. Presenting the autonomous underwater vehicles (AUV) is a good idea to bear on these problems. Utilizing AUV resolved some of the problems like divers' health risks, but some major problems still remain.

In many underwater tasks it is crucial to have a complete control and great maneuverability on the vehicle and to collect enough data to locate the vehicle as precisely as possible. All these allow moving the vehicle close to an underwater structure as closely as needed [3].

The hovering autonomous underwater vehicle (HAUV) is a novel underwater robot that combines the maneuverability of an ROV with the flexibility of autonomous operations, so as to efficiently perform detailed surveys of large marine structures such as floating vessels [4].

Lamp Ray was one of the primary commercial inspector robots which was introduced by Harris and Slate in 1999 [5]. Lamp Ray is a ROV that moves under human control.

In 2002 Trimble and Belcher designed Cetus II which using altimeters could maintain a constant distance from the hull [6].

Odyssey IV was designed in cooperation of MIT with Desset et al, which is a low cost HAUV designed for unexpected quick discovery and detection surveys and saves energy for just 1 hour [7]. One of the successful robots of this kind is the HAUV jointly developed by Bluefin Robotics and MIT which was designed particularly for underwater ship hull inspections. The goal of this design was to achieve small, low-cost robot which can be used in shallow water with great accuracy $[1,4,8]$. Negahdaripour and Firoozfam in 2005 worked on vision system of a ship hull inspector ROV [9].

One of the major efforts on underwater inspection is to improve the data collection of the robot. This can be done by using better sensors and cameras, but another issue is the trajectory of the vehicle which can take the best out of the vision sensors if planned well. Therefore, path planning plays one of the key roles in inspection of the underwater structures which is dependent on the degrees of the freedom of the vehicle. Therefore, there are numerous publications on path planning of the robots, paving the way for a more precise and efficient performance of inspection around the propellers, stern and any other particular parts with high curves. Englot and Hover in 2010 worked 
on inspection planning [10]. Similar researches are presented in [11-14].

For the first time, Gertler and Hagen in 1967 [15] developed the equation of motion for a submarine. This dynamic model has been improved through the years by Logan [16], Liang et al. [17], and Yang [18].

Designing a practical HAUV is a challenging job and requires a well-planned sequence each step of which has its particular challenges. This process starts with dynamic modeling of the vehicle, which is a difficult step because of the special physical and mechanical properties of water causing very high nonlinear behavior of the vehicle, and time varying hydrodynamic coefficients which are dependent of the vehicle shape and speed. Also, existence of unpredicted forces caused by the waves and underwater currents makes the dynamic modeling more difficult $[19,20]$. This makes engineers develop numerous control architectures which fit to each mission and cover the problem of inexact and approximate dynamic model. Most of the underwater robots are controlled by classic control method like PID controller and nowadays fuzzy controller, adoptive controller, sliding mode controller and other modern control architectures which are desirable. Yuh [21] and Carven [22] cite a range of advanced controllers used in AUVs. In the past few years, PID controller was more preferable than the other controllers. Prestero [23], Pyo et al. [24], and Choiet et al. [25] used this method owing to its advantages like being easy to maintain and apply, but it suffers from problems like being so sensitive to disturbance and parameter uncertainty and being ineffective for MIMO systems. Blasuriya and Ura [26] and Silpa-Anan and Abdallah [27] used visual servo controller designed to control the vehicle by pursuing the objects or signs in the sea, but due to short vision range in shallow water of seaports, this method does not seem practical. One of the common choices for controlling AUVs is Fuzzy logic controllers. This method is more desirable for systems with complicated dynamic model, but it is very hard to apply for the difficulties like being a time consuming process for tuning parameters [28]. This controller is mostly used to control torpedo type AUVs instead of Hovering type AUVs or HAUVs, Nag et al. [29], Ma et al. [30], Ghanavati and Ghanbarzadeh [31] used fuzzy controllers for AUVs. Sliding mode controller is a robust controller which is developed to tackle the problem of uncertainty in hydrodynamic coefficients $[32,18]$, Yoerger and Slotine [33]. Healy and Lienard [34] used this controller for torpedo type AUVs, and Arshad [35] used this method for an HAUV. In practice, application of robust control methods like sliding mode controller is difficult due to hardware limitations and implementation difficulties. Controllers designed in State space yield advantages like easy application and tune, appropriateness for MIMO systems and suitability for nonlinear systems.

\section{Dynamic Modeling of Hovering Type Autonomous Underwater Vehicle}

In this paper, in order to set up the dynamic model, we use the hydrodynamic coefficients calculated by Chin and Lau [36] and physical constants plus added mass matrix calculated by Eng et al. [37] for the RRC robot which is an open space frame ROV developed in NTU in order to inspect and maintain of underwater pipes. Dry weight of this robot is $115 \mathrm{~kg}$ and maximum depth that it can endure is $100 \mathrm{~m}$ for now. This robot is a 6 degree of freedom with 4 thrusters.

Since this paper is concerned with developing a robot with a precise and robust control, we did some modification on location and thrust direction of the thrusters on this robot, and, also, we added 2 other thrusters. Thus, we changed the dynamic of the system to reach the desirable characteristics for complex underwater inspection. As a result, we could control 5 degrees of freedom and fix the Roll by adjustment of the distance between the center of buoyancy and center of gravity; moreover, with no thruster to change this angle, we are able to produce enough self-aligning static torque. In figure 1 , we demonstrate the location and direction of each thruster; also, in figure 2, the VRML model of this robot is developed in V-realm to study the motion of the robot can be seen.

Usually, two types of coordinate system used to describe the kinematics and dynamics of the body that includes the earth-fixed frame and body-fixed reference frame that connected to the body. In figure 3, the location of each two reference frames can be observed. The coordinates system set up is the basic and first step to dynamic modeling. Body fixed frame is a Cartesian coordinate system whose positive $\mathrm{X}$ direction is along forward speed of the robot which is known as Surge, and earth fixed reference frame is also a Cartesian coordinate where distances and orientations of the robot are measured with respect of this reference frame.

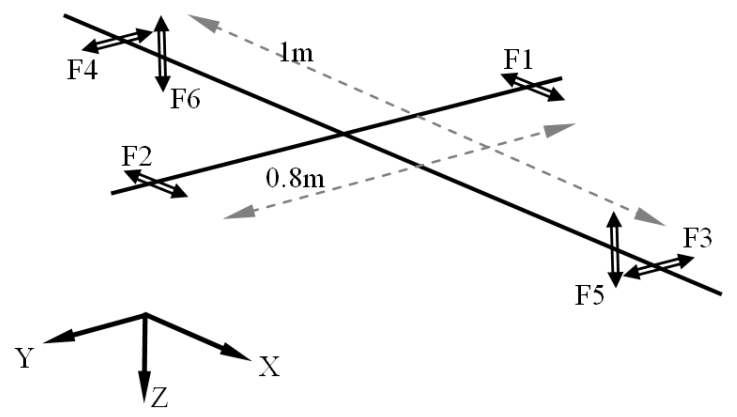

Fig. 1. Thruster's position in new HAUV

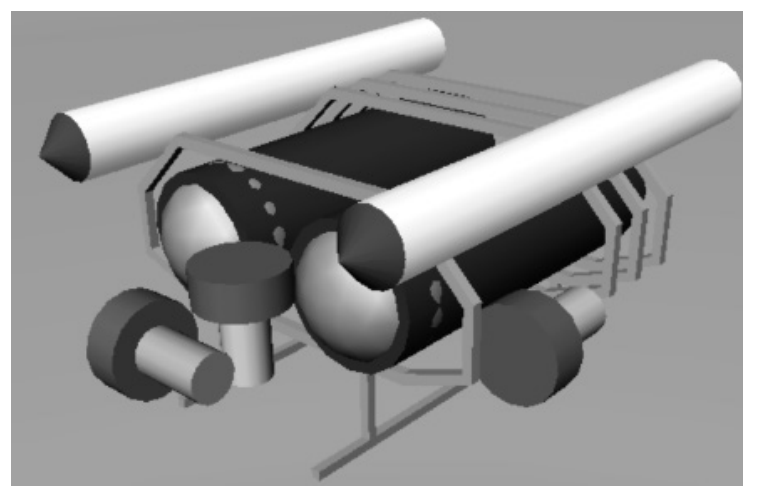

Fig. 2. Underwater robots model in V-realm 


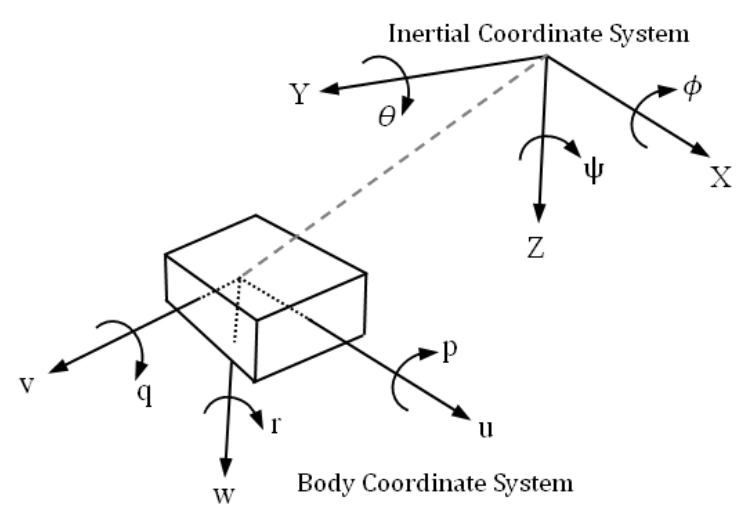

Fig. 3. Coordinate systems

With this configuration, force and moment vector can be calculated by equation (1).

$F=\left[\begin{array}{cccccc}1 & 1 & 0 & 0 & 0 & 0 \\ 0 & 0 & 1 & 1 & 0 & 0 \\ 0 & 0 & 0 & 0 & 1 & 1 \\ 0 & 0 & 0 & 0 & 0 & 0 \\ 0 & 0 & 0 & 0 & D 5 & D 6 \\ D 1 & D 2 & D 3 & D 4 & 0 & 0\end{array}\right]\left[\begin{array}{c}F 1 \\ F 2 \\ F 3 \\ F 4 \\ F 5 \\ F 6\end{array}\right]=$

$$
\left[\begin{array}{c}
F 1+F 2 \\
F 3+F 4 \\
F 5+F 6 \\
0 \\
F 5 \times D 5+F 6 \times D 6 \\
F 1 \times D 1+F 2 \times D 2+F 3 \times D 3+F 4 \times D 4
\end{array}\right]
$$

Where the parameters D1, D2...D6 are distances of thrusters $1,2 \ldots 6$ to the center of buoyancy:

$$
\begin{aligned}
& D 1=-0.4, D 2=0.4, D 3=0.5, \\
& D 4=-0.5, D 5=0.5, D 6=-0.5
\end{aligned}
$$

With respect to figure 1 and figure 3, linearized equation of motion represented by McEwen and Streitlien [38] and Radzak and Arshad [39] will become like equation (2) and equation (3). Equation (2) related to Sway and Yaw is used to design horizontal plane controller.

$$
\begin{aligned}
& {\left[\begin{array}{cccc}
m-Y_{\dot{v}} & m x_{g}-Y_{\dot{r}} & 0 & 0 \\
m x_{g}-N_{\dot{v}} & I_{z z}-N_{\dot{r}} & 0 & 0 \\
0 & 0 & 1 & 0 \\
0 & 0 & 0 & 1
\end{array}\right]\left[\begin{array}{c}
\dot{v} \\
\dot{r} \\
\dot{y} \\
\dot{\psi}
\end{array}\right]} \\
& =\left[\begin{array}{cccc}
Y_{v} U & \left(Y_{r}-m\right) U & 0 & 0 \\
N_{v} U & \left(N_{r}-m x_{g}\right) U & 0 & 0 \\
1 & 0 & 0 & U \\
0 & 1 & 0 & 1
\end{array}\right]\left[\begin{array}{c}
v \\
r \\
y \\
\psi
\end{array}\right]+
\end{aligned}
$$

$$
\left[\begin{array}{c}
F 3+F 4 \\
F 1 \times D 1+F 2 \times D 2+F 3 \times D 3+F 4 \times D 4 \\
0 \\
0
\end{array}\right]
$$

Equation (3) which is related to heave and pitch used to design vertical plane controller

$$
\left[\begin{array}{cccc}
m-Z_{\dot{w}} & -m x_{g}-Z_{\dot{q}} & 0 & 0 \\
-m x_{g}-M_{\dot{w}} & I_{y y}-M_{\dot{q}} & 0 & 0 \\
0 & 0 & 1 & 0 \\
0 & 0 & 0 & 1
\end{array}\right]\left[\begin{array}{c}
\dot{w} \\
\dot{q} \\
\dot{z} \\
\dot{\theta}
\end{array}\right]=
$$

$$
\left[\begin{array}{cccc}
Z_{w} U & \left(Z_{q}+m\right) U & 0 & 0 \\
M_{w} U & \left(M_{q}-m x_{g}\right) U & 0 & w\left(z_{b}-Z_{b}\right) \\
1 & 0 & 0 & U \\
0 & 1 & 0 & 0
\end{array}\right]\left[\begin{array}{c}
w \\
q \\
z \\
\theta
\end{array}\right]
$$

$$
+\left[\begin{array}{c}
F 5+F 6 \\
F 5 \times D 5+F 6 \times D 6 \\
0 \\
0
\end{array}\right]
$$

Next, we rewrite equations (2) and (3) in form of equation (4) which is standard form of state space representation of the system.

$$
\begin{aligned}
& \dot{x}=A x+B u \\
& y=C x+D u
\end{aligned}
$$


After we substitute hydrodynamic and physical coefficients, and also constant speed of $u=0.8 \mathrm{~m} / \mathrm{s}$ in the equations, we set up matrixes $A, B, C, D$, state vector and input vector for equation (2), so we are able to represent the system in state space form. These matrixes can be shown as:

$A=\left[\begin{array}{cccr}0.4810 & -1.4534 & 0 & 0 \\ 0 & 0.2937 & 0 & 0 \\ 1 & 0 & 0 & 0.8 \\ 0 & 1 & 0 & 1\end{array}\right]$

$B=\left[\begin{array}{cccc}0 & 0 & 0.0158 & 0.0158 \\ 0.1245 & -0.1245 & 0.1556 & -0.1556 \\ 0 & 0 & 0 & 0 \\ 0 & 0 & 0 & 0\end{array}\right]$

$C=\left[\begin{array}{llll}0 & 0 & 1 & 0\end{array}\right]$

$D=0$

$X=\left[\begin{array}{llll}v & r & y & \psi\end{array}\right]^{T}$

$u=\left[\begin{array}{llll}F 1 & F 2 & F 3 & F 4\end{array}\right]^{T}$

Also, calculated matrixes $A, B, C, D$, state vector $x$ and input vector $u$ for equation (3) are shown in following:

$$
\begin{aligned}
& A=\left[\begin{array}{cccc}
2.5722 & 4.0800 & 0 & 0 \\
0 & 0.3490 & 0 & -33.7927 \\
1 & 0 & 0 & -0.8 \\
0 & 1 & 0 & 1
\end{array}\right] \\
& B=\left[\begin{array}{cc}
0.0443 & 0.0443 \\
0.1498 & -0.1498 \\
0 & 0 \\
0 & 0
\end{array}\right] \\
& C=\left[\begin{array}{llll}
0 & 0 & 1 & 0
\end{array}\right] \\
& D=0 \\
& x=\left[\begin{array}{llll}
w & q & z & \theta
\end{array}\right]^{T} \\
& u=\left[\begin{array}{ll}
F 5 & F 6
\end{array}\right]^{T}
\end{aligned}
$$

At this point, we calculated state space representation of the system in vertical and horizontal plane, so we are able to design the controller in the next step.

\section{Control Architecture}

The current controller is designed by pole placement method with thruster forces as input vector in state space. Firstly, an algorithm is developed in Matlab to design the state feedback controller upon the linearized system, and then the controller is applied to linearized system and nonlinear system. To control the forward speed of the robot, PD controller is used. Figure 3 demonstrates the block diagram of the system and controller.

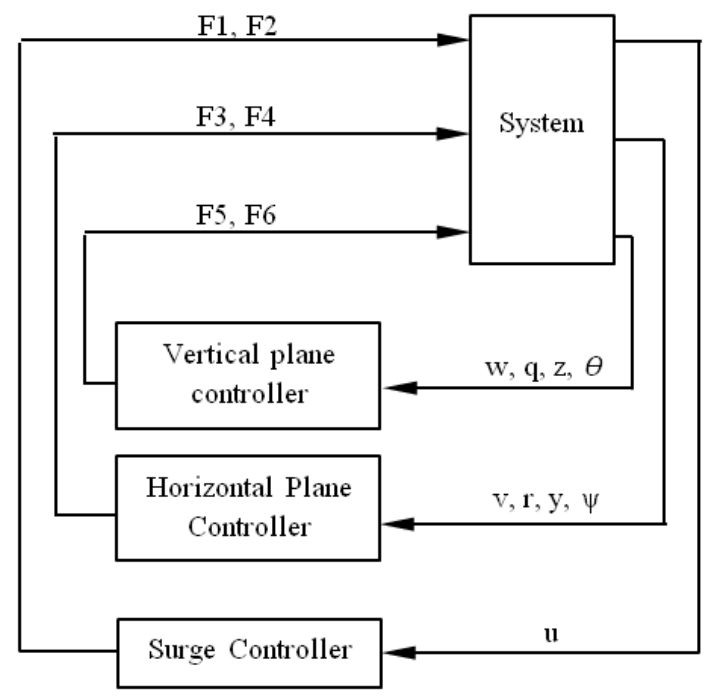

Fig. 4. Block diagram of the system and controller

\section{Results and Discussion}

The controller is designed and tested on the linearized model. Figure 5 shows a spiral maneuver which has been designed to take the controller to its limitation. This maneuver is obtained by giving a sine wave input to both vertical and horizontal controllers, and this wave has amplitude of 2.5 meter and a frequency of $28 \mathrm{deg} / \mathrm{s}$. As shown in figure 5, the robot is able to accomplish the task precisely. Figure 6 demonstrates the force output of thrusters 3, 4, 5 and 6. In linearized model, the forward speed is constant, and its value is directly involved in the dynamic model. Therefore, thrusters 1 and 2 have not been involved in the moving, and they are not considered in this simulation.

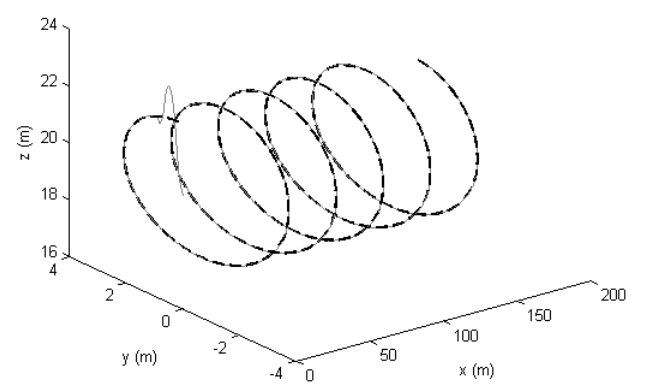

Fig. 5. Linear model response to desired helical path 

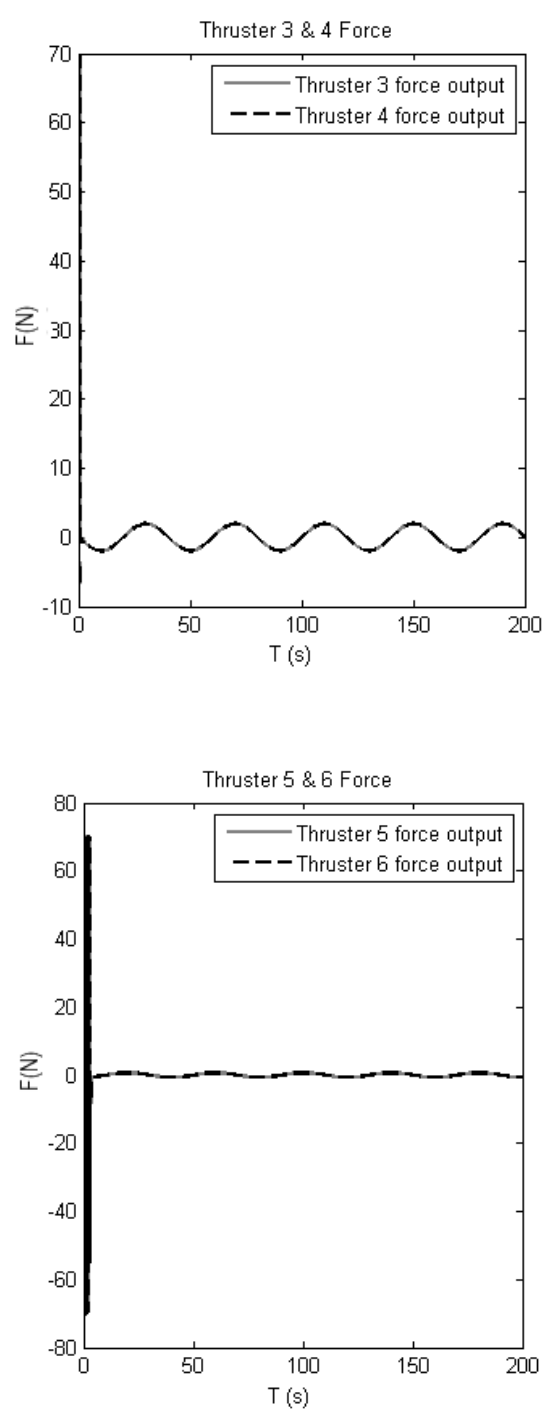

Fig. 6. Control inputs in the linear model simulation

After the validation of the controller on the linear model, the controller has been implemented to the non-linear model. Same path as figure 5 has been given to the underwater robot, so we could compare and analyze the results. Figure 7 shows the desirable path and the robot path for this maneuver. In figure 8, you can see the output forces of the thrusters.

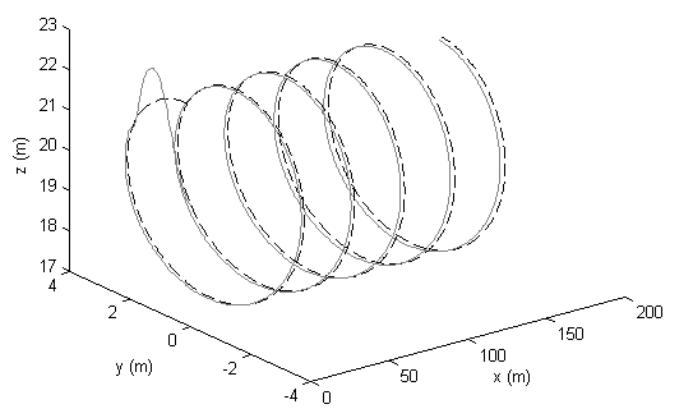

Fig. 7. Nonlinear model response to desired helical path
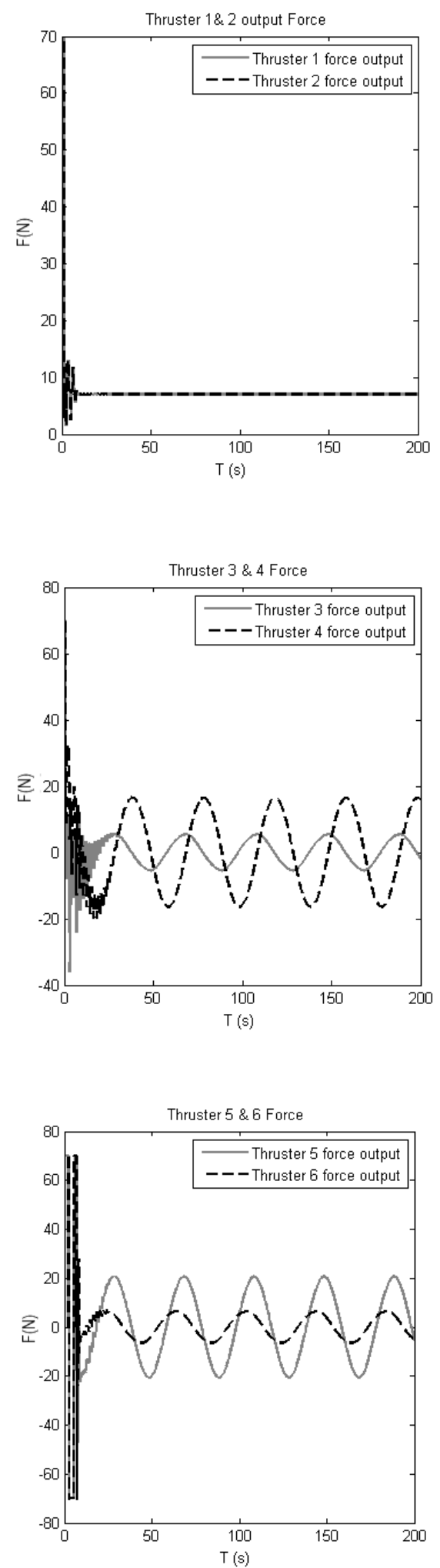

Fig. 8. Control inputs in the nonlinear model simulation

When an organization utilizes AUVs for underwater inspection, highest possibility of control on the vehicle is desirable. It is sometimes very useful and demanding to have a complete control on the an- 
gles of orientation of the vehicle. In figures 9 and 10, a maneuver is presented in which two states are controlled at the same time. $\mathrm{Y}$ and $\theta$ degrees of freedom which represent lateral movement and rotation along y axis simultaneously are controlled. Such a maneuver allows for better 3D model developing, especially around the complex shaped parts like propeller of the ship or sonar dome; moreover, when a welding mission is expected, having such capability plays a key role. Figures 11 show the thrust outputs of thrusters for this maneuver.

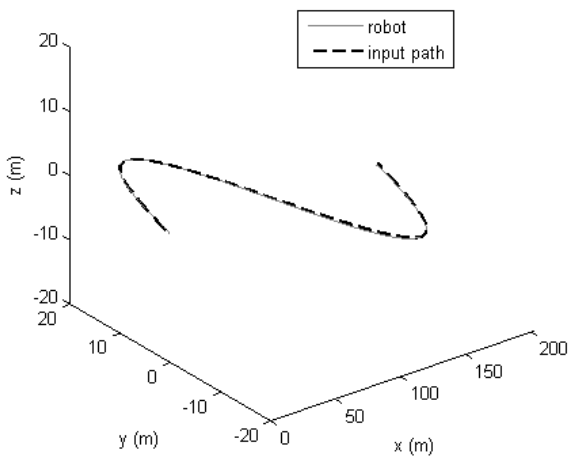

Fig. 9. Sinusoidal maneuver along y axis simultaneous with $\theta$ control

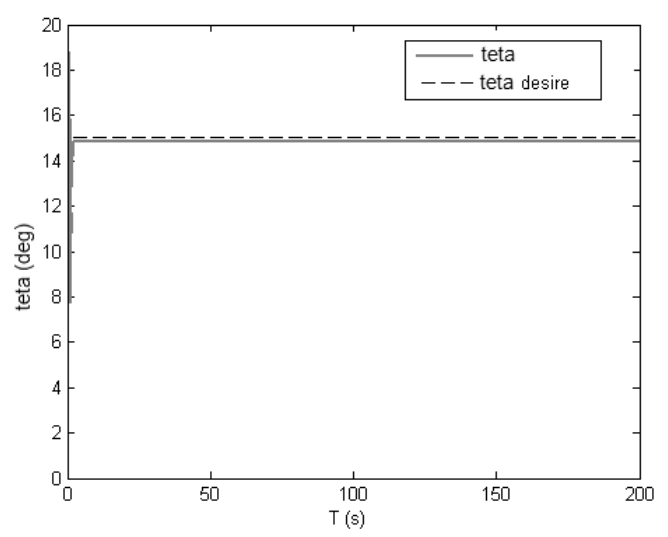

Fig. 10. $\theta$ control simultaneous with y control

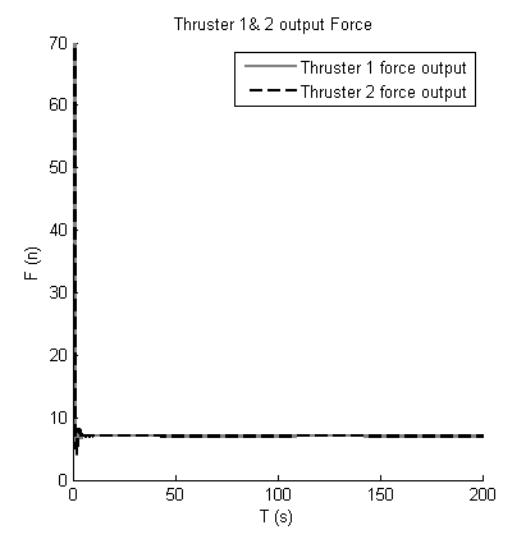

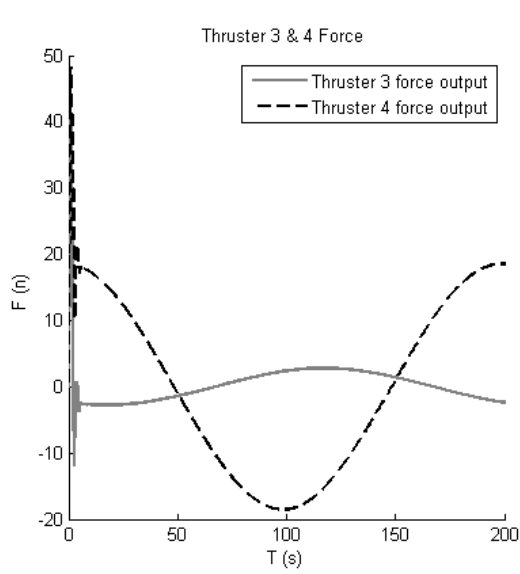

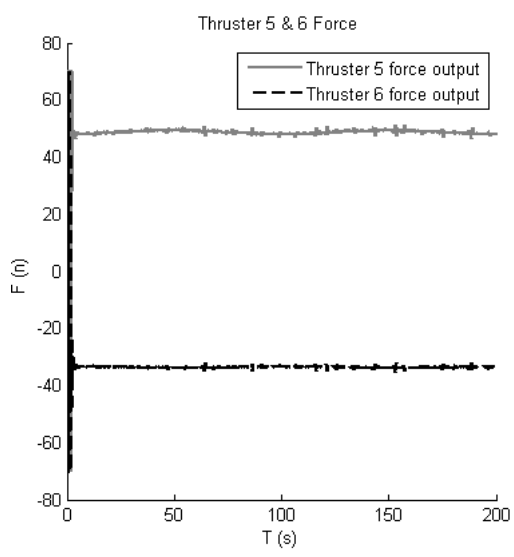

Fig. 11. Control inputs for the simultaneous control of $\theta$ and $y$

Figures 12 and 13 are showing the results of controlling degrees of freedom $\mathrm{z}$ and $\theta$ at the same time, figure 12 represents 3D path of the robot and figure 13 represents controlling pitch at a fixed angle in this maneuver. As the robot increases depth up to 50 meters, the pitch angle will stay fixed at 20 degrees. This maneuver is more challenging than the previous one, since when the degrees of freedom y and $\theta$ are controlled, two different controllers on different planes are controlling the robot, that's mean thrusters 4 and 5 are controlling the lateral movement and thrusters 5 and 6 are controlling the $\theta$ angle, but while controlling the $\mathrm{z}$ and $\theta$, both degrees of freedom must be controlled by thrusters 5 and 6 , which exist at vertical plane controller. As the result shows, the controller is capable of accomplishing this maneuver accurately. Figures 14 show the thrust outputs of thrusters for this maneuver 


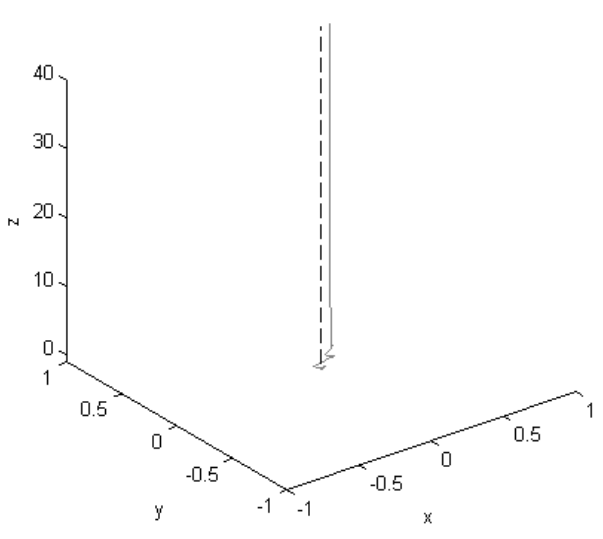

Fig. 12. $z$ control simultaneous with $\theta$ control

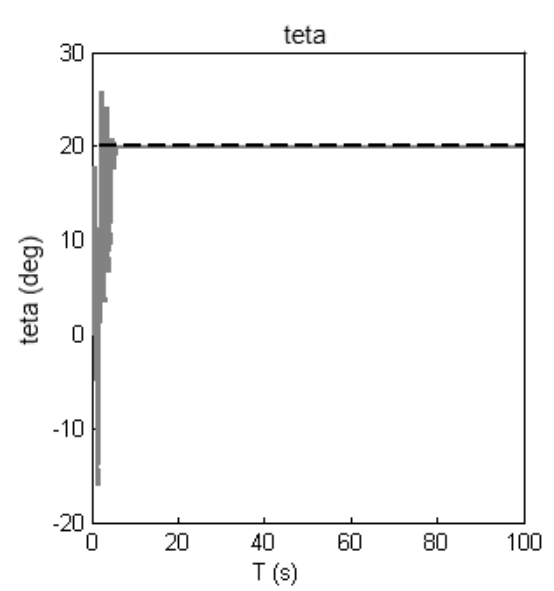

Fig 13. $\theta$ control simultaneous with $\mathrm{z}$ control
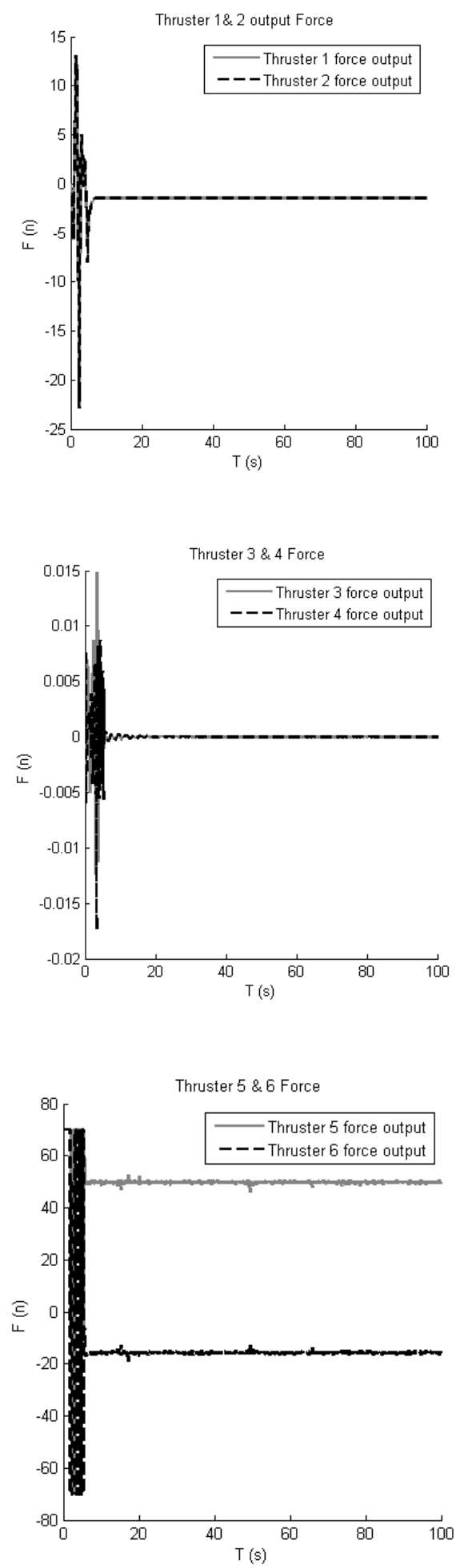

Fig. 14. Control inputs for the simultaneous control of $\theta$ and $z$ 
The next goal is to control y and $\psi$ together. This is a very challenging maneuver to control these two degrees of freedom, because when thrusters 3 and 4 are controlling the lateral position of the vehicle at the same time, thrusters 1 and 2 are controlling both forward speed (surge) and yaw angle. This task has been done by using an algorithm that is developed to switch between the controllers any time that the error exceeds the allowed value. Figures 15 and 16 are showing this maneuver; at the same time, thrusters 5 and 6 are still able to control vehicle depth. This means that, in these maneuvers, three states are controlled simultaneously.

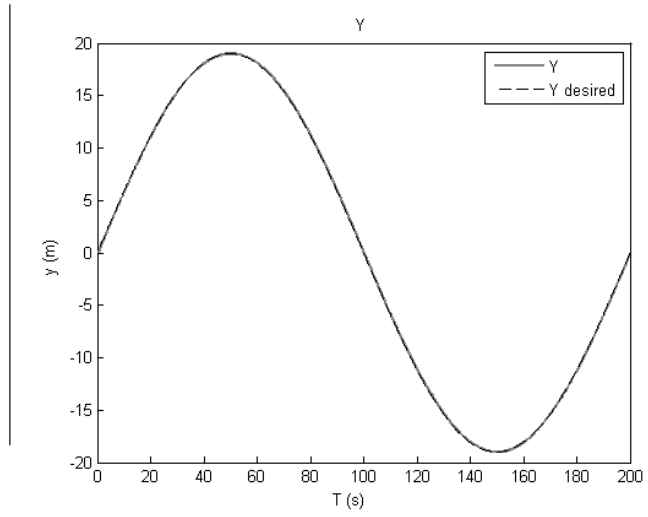

Fig. 15. y control simultaneous with $\psi$ control

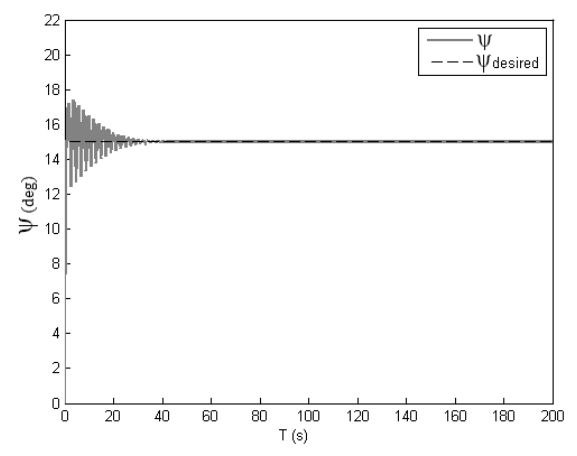

Fig. 16. $\psi$ control simultaneous with y control
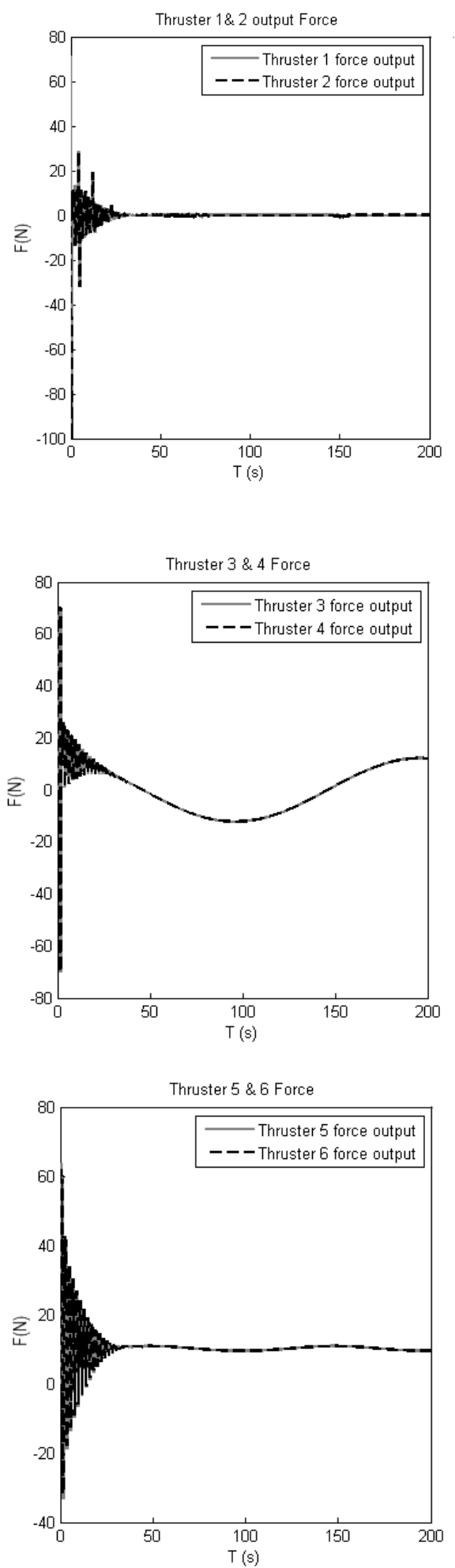

Fig. 17. Control inputs for simultaneous control of $y$ and $\psi$ 


\section{Robustness Examination}

To examine the robustness of the controller, we targeted two important parts: a) uncertainty of hydrodynamic coefficients and added mass, b) external disturbances

A certain percentage of error is expected in estimation of hydrodynamic coefficient and added mass, so we multiplied this coefficient to a certain factor we named uncertainty factor in the non-linear system. In this paper, the uncertainty factor of 2 is applied to the hydrodynamic coefficients and added mass parameters.

To test the controller against disturbance, three constant but different forces were applied to the system along $\mathrm{x}, \mathrm{y}$ and $\mathrm{z}$ directions at a certain period of time (figure 18).

For full robustness examination, a simulation has been utilized in which both parameters uncertainty and external disturbances are applied to the system. The desired path is the same one as figure 5 .

Figure 19 presents a trajectory of underwater robot under these conditions. The result shows, despite these severe conditions, the new underwater robot and presented controller acceptably follow the desired path. Figures 20 show the thrust outputs of thrusters for these conditions.

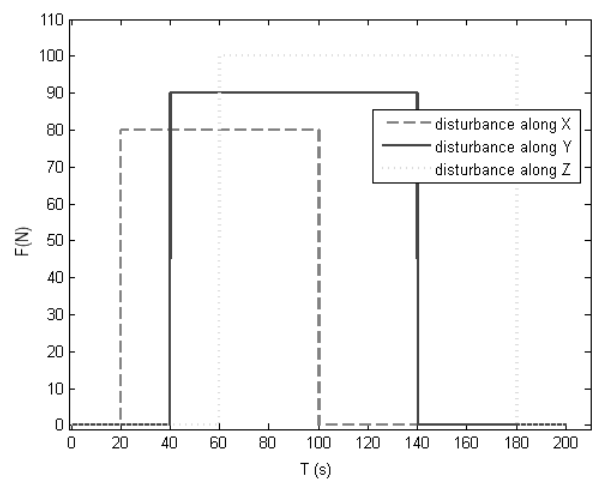

Fig. 18. External disturbance as forces

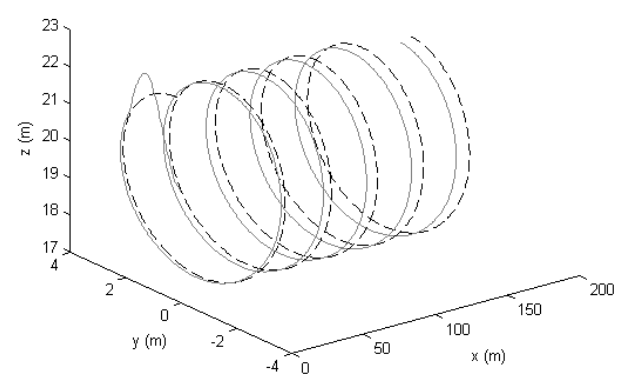

Fig. 19. Tracking of desired path under external disturbance and parameter uncertainty
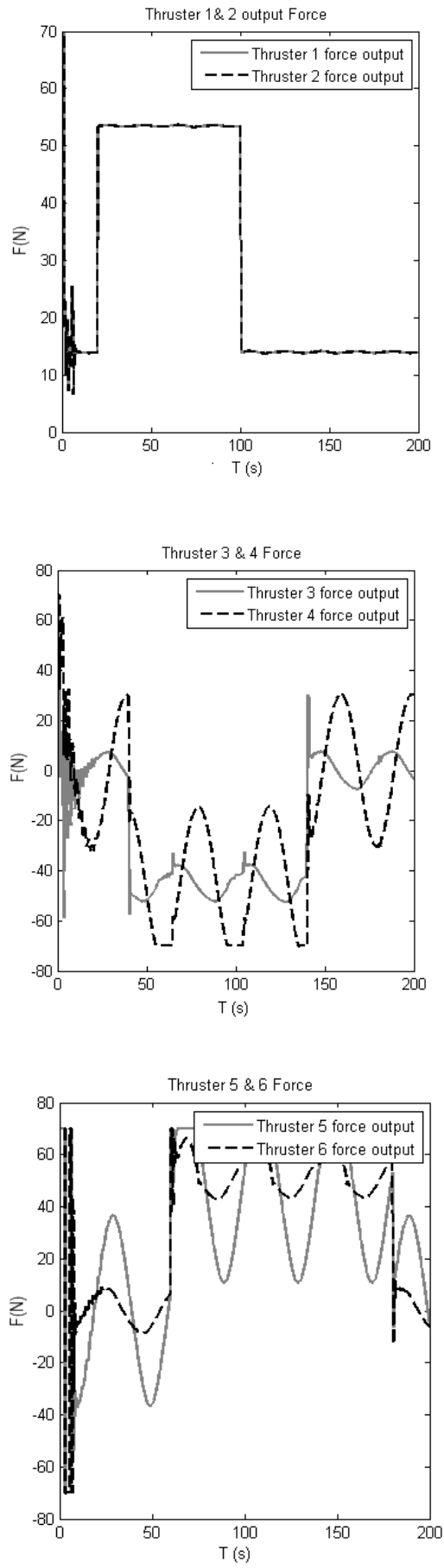

Fig. 20. Control inputs under external disturbance and parameter uncertainty

\section{Conclusion}

In this paper a highly maneuverable and controllable underwater vehicle was presented. The designed controller was able to control this multi-input, multioutput system so that the robot could track the complex trajectory. The control of multi state variables is a consequence of these presented underwater vehicle and controllers. All these characteristics cause that this underwater robot is a very suitable and practical choice for missions such as: underwater inspections (pipes, ship hull, dams, oil platforms ...), rescue mission, welding and generally any mission which requires a quite maneuverable and hover capable robot. The underwater environment is very complex with 
serious disturbance problems; therefore a robust controller is required for underwater robot; so in this paper, presented controller was tested to be robust.

\section{AUTHORS}

Faryar Shamshiri Amirkolai - Mechanical Engineering, Hakim Sabzevari University, Sabzevar, Iran.

Reza Hasanzadeh Ghasemi* - Mechanical Engineering, Hakim Sabzevari University, Sabzevar, Iran, Email: r.h.ghasemy@gmail.com.

* Corresponding author

\section{REFERENCES}

[1] F. Hover, J. Vaganay, M. Elkins, S. Willcox, V. Polidoro, J. Morash and others, "A vehicle system for autonomous relative survey of in-water ships", Mar. Technol. Soc. J., vol. 41, no. 2, 2007, 44-55, 10.4031/002533207787442196.

[2] U. C. Pati, 3D Surface Geometry and Reconstruction: Developing Concepts and Applications, $1^{\text {st }}$ edition, IGI Global, 2012.

[3] F. Shamshiri Amirkolai and R. Hasanzadeh Ghasemi, "Representation of an Autonomous Underwater Vehicle and Trajectory Controller design for in-water ship hull inspection", Modares Mechanical Engineering, vol. 15, no. 10, 2016, 12-22 (In Persian).

[4] J. Vaganay, M. Elkins, D. Esposito, W. O’ Halloran, F. Hover and M. Kokko, "Ship hull inspection with the HAUV: US Navy and NATO demonstrations results". In: Proc. of IEEE/MTS OCEANS Conference, 2006, 1-6, 10.1109/OCEANS.2006.307039.

[5] S. E. Harris and E. V. Slate, "Lamp Ray: ship hull assessment for value, safety and readiness". In: Proc. of IEEE/MTS Oceans '99. Riding the Crest into the 21st Century. Conference and Exhibition., vol. 1, 1999, 493-500, 10.1109/OCEANS. 1999.799792.

[6] G. M. Trimble and E. Belcher, "Ship berthing and hull inspection using the CetusII AUV and MIRIS high-resolution sonar". In: Proc. of IEEE/MTS OCEANS Conference, vol. 2, 2002, 1172-1175, 10.1109/OCEANS.2002.1192132.

[7] S. Desset, R. Damus, F. Hover, J. Morash and V. Polidoro, "Closer to deep underwater science with ODYSSEY IV class hovering autonomous underwater vehicle (HAUV)". In: Europe Oceans 2005, vol. 2, 2005, 758-762, 10.1109/OCEANSE. 2005.1513151.

[8] J. Vaganay, M. Elkins, S. Willcox, F. Hover, R. Damus, S. Desset and others, "Ship hull inspection by hull-relative navigation and control". In: Proc. of IEEE/MTS OCEANS Conference, vol. 1, 2005, 761-766, 10.1109/OCEANS.2005.1639844.

[9] S. Negahdaripour and P. Firoozfam, "An ROV stereovision system for ship-hull inspection", IEEE J. Oceanic Eng., vol. 31, no. 3, 2006, 551-564, 10.1109/JOE.2005.851391.

[10] B. Englot and F. Hover, "Inspection planning for sensor coverage of 3D marine structures". In: Proc. of IEEE/RSJ International Conference, Intelligent, Robots and Systems, 2010, 4412-4417, 10.1109/IROS.2010.5648908.

[11] B. Englot and F. S. Hover, "Sampling-Based Coverage Path Planning for Inspection of Complex Structures". In: Proc. International Conference of Automated Planning and Scheduling, 2012, 29-37.

[12] G. A. Hollinger, B. Englot, F. Hover, U. Mitra and G. S. Sukhatme, "Uncertainty-driven view planning for underwater inspection". In: Proc. of IEEE International Conference on Robotics and Automation, 2012, 4884-4891, 10.1109/ ICRA.2012.6224726.

[13] G. A. Hollinger, B. Englot, F. S. Hover, U. Mitra and G. S. Sukhatme, "Active planning for underwater inspection and the benefit of adaptivity", Int. J. Robot. Res., vol. 32, no. 1, 2012, 3-18, 10.1177/0278364912467485.

[14] F. S. Hover, R. M. Eustice, A. Kim, B. Englot, H. Johannsson, M. Kaess and others, "Advanced perception, navigation and planning for autonomous in-water ship hull inspection", Int. J. Robot. Res., vol. 31, no. 12, 2012, 1445-1464, $10.1177 / 0278364912461059$.

[15] M. Gertler and G. R. Hagen, "Standard equations of motion for submarine simulation", Defense Technical Information Center, Fort Belvoir, VA, USA, 1967, 10.21236/AD0653861.

[16] C. L. Logan, "A comparison between H-infinity/mu-synthesis control and sliding-mode control for robust control of a small autonomous underwater vehicle". In: Proc. of the Symposium on Autonomous Underwater Vehicle Technology, 1994, 399-416, 10.1109/ AUV.1994.518653.

[17] X. Liang, J. Zhang, Y. Qin and H. Yang, "Dynamic Modeling and Computer Simulation for Autonomous Underwater Vehicles with Fins", J. Comput. (Taipei), vol. 8, no. 4, 2013, 1058-1064.

[18] C. Yang, "Modular modeling and control for autonomous underwater vehicle (AUV)", M.S. Thesis, Mechanical Enigineering, National University of Singapore, Singapore, 2008.

[19] F. S. Amirkolai and R. Hasanzadeh Ghasemi, "Designing a Trajectory controller in State Space for a Hovering type Autonomous Under- 
water Vehicle". In: 6th International Offshore Industies Conference, 2015 (In Persian).

[20] F. S. Amirkolai and R. Hasanzadeh Ghasemi, "Designing a discrete controller for an Autonomous Underwater Vehicle under sensoring malfunction". In: 16th Marine Industries Conference, 2014 (In Persian).

[21] J. Yuh, "Design and control of autonomous underwater robots: A survey", Auton. Robots, vol. 8, no. 1, 2000, 7-24, 10.1023/A:1008984701078.

[22] P. J. Craven, R. Sutton and R. S. Burns, “Control strategies for unmanned underwater vehicles", J. Navig., vol. 51, no. 1, 1998, 79-105, 10.1017/ S0373463397007601.

[23] T. Prestero, "Verification of a six-degree of freedom simulation model for the REMUS autonomous underwater vehicle", M.S. Thesis, Massachusetts Institute of Technology and Woods Hole Oceanographic Institution, Woods Hole, MA, USA, 2001.

[24] J. Pyo, H. G. Joe, J. H. Kim, A. Elibol and S. C. Yu, "Development of Hovering-Type AUV "cyclops" for Precision Observation". In: Proc. of OCEANS, 2013, 1-5, 10.23919/OCEANS.2013.6741060.

[25] H. T. Choi, A. Hanai, S. K. Choi and J. Yuh, "Development of an underwater robot, ODIN-III". In: Proc. 2003 IEEE/RSJ International Conference on Intelligent Robots and Systems, vol. 1, 2003, 836-841, 10.1109/IROS.2003.1250733.

[26] A. Balasuriya and T. Ura, "Underwater cable following by Twin-Burger 2". In: Proc. 2001 ICRA. IEEE International Conference on Robotics and Automation, vol. 1, 2001, 920-925, 10.1109/ ROBOT.2001.932668.

[27] C. Silpa-Anan, S. Abdallah and D. Wettergreen, "Development of autonomous underwater vehicle towards visual servo control". In: Proc. of the Australian Conference on Robotics and Automation, 2000, 105-110.

[28] L. G. García-Valdovinos, T. Salgado-Jiménez, M. Bandala-Sánchez, L. Nava-Balanzar, R. Hernández-Alvarado and J. A. Cruz-Ledesma, "Modelling, Design and Robust Control of a Remotely Operated Underwater Vehicle", Int. J. Adv. Robot. Syst., vol. 11, no. 1, 2014, 10.5772/56810.

[29] A. Nag, S. S. Patel and S. Akbar, "Fuzzy logic based depth control of an autonomous underwater vehicle". In: Proc. of International Multi Conference on Automation, Computing, Communication, Control and Compressed sensing, 2013, 117-123, 10.1109/iMac4s.2013.6526393.

[30] S. Ma, Y.-J. Pang, T.-D. Zhang and C. Lv, "Fuzzy S plane controller for motion control of underwater vehicles". In: 2011 6th IEEE Conference on Industrial Electronics and Applications, 2011, 1640-1645, 10.1109/ICIEA.2011.5975853.

[31] M. Ghanavati and A. Ghanbarzadeh, "Control and Guidance of an Underwater Robot via Fuzzy Control Method", International Journal of Advanced Design and Manufacturing Technology, vol. 4, no. 1, 2010, 25-32.

[32] L. A. Cooney, "Dynamic response and maneuvering strategies of a hybrid autonomous underwater vehicle in hovering", M.S. Thesis, Massachusetts Institute of Technology, USA, 2009.

[33] D. R. Yoerger and J.-J. Slotine, "Robust trajectory control of underwater vehicles", IEEE J. Oceanic Eng., vol. 10, no. 4, 1985, 462-470, 10.1109/ JOE.1985.1145131.

[34] A. J. Healey and D. Lienard, "Multivariable sliding mode control for autonomous diving and steering of unmanned underwater vehicles", IEEE J. Oceanic Eng., vol. 18, no. 3, 1993, 327-339, 10.1109/JOE.1993.236372.

[35] M. R. Arshad and M. Y. Radzak, "Design and development of an autonomous underwater vehicle test-bed (USM-AUV I)". In: ICARCV 2004 8th Control, Automation, Robotics and Vision Conference, 2004., vol. 1, 2004, 257-260, 10.1109/ ICARCV.2004.1468833.

[36] C. Chin and M. Lau, "Modeling and testing of hydrodynamic damping model for a complex-shaped remotely-operated vehicle for control", Journal of Marine Science and Application, vol. 11, no. 2, 2012, 150-163, 10.1007/s11804-0121117-2.

[37] Y. Eng, M. Lau and C. Chin, "Added mass computation for control of an open-frame remotely-operated vehicle: Application using WAMIT and MATLAB", J. Mar. Sci. Technol., vol. 22, no. 2, 2013, 1-14, 10.6119/JMST-013-0313-2.

[38] R. McEwen and K. Streitlien, "Modeling and Control of a Variable-Length AUV". In: 12th International Symposium on Unmanned Untethered Submersible Technology (UUST) Conference, 2001.

[39] M. Radzak and M. Arshad, "AUV Controller Design and Analysis Using Full-State Feedback". In: 9th WSEAS International Conference on Systems, Wisconsin, 2005. 